\title{
Histopathological and Immunohistochemical Study of the Distinction between Oral Lichen Planus and Oral Lichenoid Lesions
}

\author{
Takehiro Suzuki', Masaaki Suemitsu ${ }^{2,3}$, Mitsuko Nakayama ${ }^{3}$, Chieko Taguchi ${ }^{4}$,

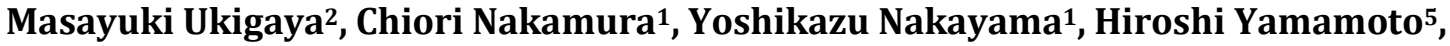 \\ Kayo Kuyama ${ }^{2,3^{*}}$
}

${ }^{1}$ Nihon University Graduate School Dentistry at Matsudo, Oral Pathology, Chiba, Japan

${ }^{2}$ Department of Pathological Diagnosis, Nihon University Hospital at Matsudo, Chiba, Japan

${ }^{3}$ Department of Pathology, Nihon University School of Dentistry at Matsudo, Chiba, Japan

${ }^{4}$ Department of Community Oral Health, Nihon University School of Dentistry at Matsudo, Chiba, Japan

${ }^{5}$ Department of Oral Surgery, Nihon University School of Dentistry at Matsudo, Chiba, Japan

Email: ${ }^{\star}$ kuyama.kayo@nihon-u.ac.jp

How to cite this paper: Suzuki, T., Suemitsu, M., Nakayama, M., Taguchi, C., Ukigaya, M., Nakamura, C., Nakayama, Y., Yamamoto, H. and Kuyama, K. (2021) Histopathological and Immunohistochemical Study of the Distinction between Oral Lichen Planus and Oral Lichenoid Lesions. Open Journal of Stomatology, 11, 91-106. https://doi.org/10.4236/ojst.2021.112008

Received: December 28, 2020

Accepted: February 22, 2021

Published: February 25, 2021

Copyright $\odot 2021$ by author(s) and Scientific Research Publishing Inc. This work is licensed under the Creative Commons Attribution International License (CC BY 4.0).

http://creativecommons.org/licenses/by/4.0/

(c) (i) Open Access

\begin{abstract}
Background: Oral potentially malignant disorders, which include oral lichen planus (OLP), are clinical presentations that carry a risk of development to cancer in the oral cavity. Oral lichenoid lesions (OLLs) are also termed interface/lichenoid mucositis. Malignant transformation of them remains controversial, but distinct clinical and histological criteria for how to differentiate OLP from OLLs have not been developed. Objectives: The purpose of this study was to elucidate findings that can allow histopathological differentiation of OLP and OLLs using histomorphological and immunohistochemical analyses. Materials and Methods: Analyses were performed in 10 cases diagnosed with OLP and 9 cases diagnosed with OLLs. Cytokeratin 19 (CK19), Ki-67 and CD3 were used as primary antibodies to detect basal cells, proliferative activity and T-cell distribution, respectively, and Perlecan and COX-2 to evaluate epithelial intracellular arrangements and interstitial distributions of proteoglycans and enzymes. Results: For CK19, positive cells were significantly found in OLLs at both the prominent area and site adjacent to the lesion comparison with those of OLP's. The number of COX-2 positive cells was significantly higher in spinous and basal layers in OLLs of the prominent area. Additionally, OLLs showed mild to moderate expression for perlecan in the basal to spinous layers and in subepithelial tissue. Conclusion: Almost no basal cells were noted in the prominent area in OLP. COX-2 and perlecan were found in the basal to spinous layers in OLLs. Although there are restric-
\end{abstract}


tions, these suggested the possibility of helping to distinguish between OLP and OLLs.

\section{Keywords}

Oral Lichen Planus (OLP), Oral Lichenoid Lesions (OLLs), Immunohistochemical Staining, CK19, COX-2

\section{Introduction}

Oral lichen planus (OLP) is a chronic inflammatory disease involving abnormal keratinization of the oral mucosa [1] [2]. Several clinical types of OLP have been described. The most common is the reticular form, involving the buccal mucosa with symmetrical lesions, characterized by numerous interlacing white keratotic lines or striae (so-called Wickham's striae) and few symptoms [1]. In addition, OLP has histopathologically demonstrated hyperkeratosis or parakeratosis, a saw-tooth profile of the rete ridges, liquefaction degeneration of the basal cell layer, and compact and band-like lymphocytic (predominantly T-cell) infiltration of the epitheliomesenchymal junction [3]. As mentioned above, although OLP is diagnosed by combining gross and histopathological findings, concordance rates are low compared to other oral mucosal diseases [4]. Our previous study proposed oblique running of papillary loops to white striae as a histopathological finding allowing definitive diagnosis of OLP, because this finding is observed regardless of the stage [4]. In addition, the basal cell layer is lost due to liquefaction degeneration and lymphocytes were reportedly in contact with the spinous layer [4]. Various histopathological reports have provided descriptions of the basal cell layer in OLP, but no consensus has yet been reached [5] [6] [7].

On the other hand, clinically and histopathologically similar cases have been distinguished as oral lichenoid lesions (OLLs) in the oral mucosa. Differentiation between OLP and OLLs is extremely difficult unless the cause of the pathological condition can be identified [8]. OLLs appear clinically similar to OLP, and also present with liquefaction degeneration of the basal cell layer and an appearance of civatte bodies histopathologically [9].

In 2017, precancer lesions and precancer conditions were combined to create the new concept of oral potentially malignant disorders (OPMDs), which included OLP [10]. OPMDs are clinical presentations that carry a risk of development to cancer in the oral cavity [10]. OLLs are also termed interface mucositis or lichenoid mucositis [11]. Malignant transformation of these lesions remains controversial, but distinct clinical and histological criteria for how to differentiate OLP from OLLs have not been developed [11].

The purpose of this study was thus to elucidate those findings that can allow histopathological differentiation of OLP and OLLs using histomorphological and immunohistochemical analyses. 


\section{Materials and Methods}

\subsection{Subjects}

Histopathological, and immunohistochemical evaluations were performed in 10 cases ( 5 males, 5 females) diagnosed with OLP and 9 cases ( 4 males, 5 females) diagnosed with OLLs. Subjects were selected from the pathology files of the Department of Oral Pathology in Nihon University School of Dentistry at Matsudo from 2000 to 2019 . Mean age was $50.3 \pm 11.4$ years in OLP and $63.8 \pm 14.0$ years in OLLs. Subject characteristics in this study are summarized in Table 1. Cases diagnosed as OLP or OLLs by 3 dentists (including an oral surgeon) were based on gross findings of surface shape, background, and intraoral pictures of the lesion described in the electronic medical records. The conditions for the case selection of the present study are shown. At first, complete macroscopic view records were available for all patients, along with dental history of the lesion and a biopsy specimen comprising an area with epithelium and submucosal tissue. As for OLP, the cases were of unknown cause [9]. Meanwhile OLLs were considered as cases with a cause, such as, drugs, dental restorative materials, GVHD and other associated factors [9]. Patients with a history of exposure to dental materials, drugs [12], any treatment for lichen planus or drugs associated with lichenoid reaction before biopsy, any malignant or viral involvement in the mouth and pregnant women were excluded from the study sample.

Macroscopic findings were reconfirmed and classified by oral surgeons for 19 patients with well-defined looping and intersecting white lines/striae/patches with or without erosions and ulcerations [13] [14] [15] in the present study. For both lesions, clinical inspection classifications for the most prominent site were made independently by 2 oral surgeons and 3 oral pathologists based on the 6 types described by Andreasen [16] and the 2 types defined by Brant [17]. In cases of disagreement, classifications were discussed in a joint session until a consensus was reached. Biopsy specimens were obtained for all 19 patients by oral surgeons, sampling an area at the boundary of the most prominent lesion and an adjacent area of normal mucosa.

\subsection{Histological Preparation}

Histological specimens of OLP were stained using hematoxylin and eosin and selected by the following definitive histopathological criteria according to the American Academy of Oral and Maxillofacial Pathology: 1) presence of a well-defined band-like zone of cellular infiltration consisting mainly of lymphocytes in the superficial part of the connective tissue; and 2) signs of "liquefaction degeneration" in the basal layer; and 3) absence of epithelial dysplasia [18]. Conversely, specimens of OLLs stained by HE were selected by the criteria described in previous reports [9] [18]: 1) presence of associated factors; 2) diffuse and deeper distribution of lymphocytes; 3) no degeneration of basement membrane; and 4) no vascularity in basal cells. Patients with lesions that did not reflect the above strict histological criteria were not included in the study. Cases 


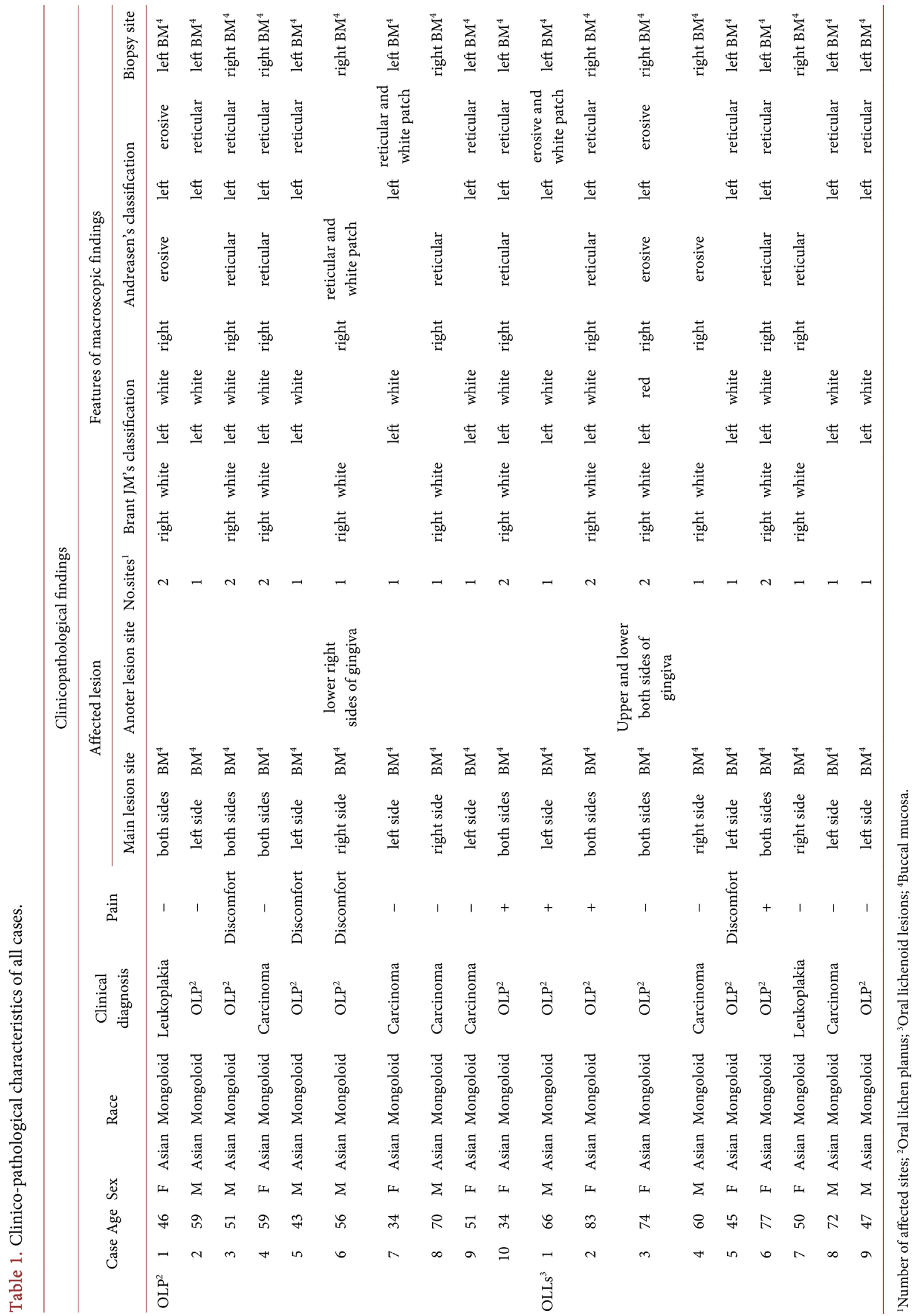


accompanied by secondary inflammation with ulcer/erosion and evidence of malignancy were also excluded. The normal oral mucosae were obtained through polypectomy from perilesional areas of fibroma without epithelial dysplasia to constitute the control group ( 2 males, 1 female; 3 samples of buccal mucosae). Four oral pathologists blinded to the biopsy material made the histopathological diagnosis of OLP and OLLs, and the assessments of immunohistochemical staining. Histopathological and immunohistochemical evaluations were identical among the 4 oral pathologists. Nineteen specimens underwent Papanicolaou staining.

\subsection{Immunohistochemical Preparation}

Immunohistochemical studies were conducted using $10 \%$ neutral formalin solution-fixed, paraffin-embedded tissues from all cases. Five serial sections (each 4 $\mu \mathrm{m}$ thick) were prepared and deparaffinized in xylene and hydrated in graded ethanol solution for further immunohistochemical analysis to calculate microvascular irregularities. The EnVision + Polymer System (Dako, Glostrup, Denmark), which also carries secondary antibody molecules, was used for antigen detection. Cytokeratin 19 (CK19, RCK108, 1:50; DakoCytomation, Glostrup, Denmark), Ki-67 (MIB-5, 1:50; DakoCytomation) and CD3 (F7.2.38, 1:50; DakoCytomation) were used as primary antibodies to detect basal cells, proliferative activity and T-cell distribution, respectively. Perlecan (received from Prof. Saku, Osaka Dental University) [19] [20] and COX-2 (CX-294, 1:100; DakoCytomation) to evaluate epithelial intracellular arrangements and interstitial distributions of proteoglycans and enzymes. Sections were developed in a solution of 3,3'-dianibobenzidine tetrahydrochloride. Finally, all sections were counterstained with Mayer's hematoxylin. Inflammatory oral mucosa and epithelial dysplasia were used as positive controls for primary antibodies. To evaluate the immunohistochemical staining technique, mouse and rabbit universal g-negative controls (DakoCytomation) were used as negative controls during the staining procedure instead of primary antibodies. Slides were examined under light microscopy and projected on a color monitor. All specimens of an area at the boundary part of the most prominent lesion and an adjacent area of normal mucosa were randomly photographed with $\geq 5$ fields of view $(\times 40)$, positive reactions to the various antibodies were evaluated. The thicknesses of the cornified, spinous and basal layers were measured in Papanicolaou-stained specimens. A digital camera for the microscope was used to obtain images, and CellSens Standard software (OLYMPUS, Tokyo, Japan) was used for image analysis.

\subsection{Statistical Assessment}

All statistical analyses were performed using SPSS for Windows version 14. OJ (IBM, Tokyo, Japan). Statistical analyses for positive rates of CK19, Ki-67 and COX-2 were performed using the Mann-Whitney $U$ test. Comparative analyses for average thicknesses of each layer were performed using Friedman's test and 
Scheff's test. Values of $p<0.05$ were considered significant.

\subsection{Compliance with Ethical Standards}

Informed consent was obtained from all individuals included in the study. All procedures performed in studies involving human participants were in accordance with the ethical standards of the Committee on Studies Involving Human Beings of Nihon University School of Dentistry at Matsudo (EC-18-15-14-2) and with the 1964 Declaration of Helsinki and its later amendments or comparable ethical standards.

\section{Results}

\subsection{Clinicopathological Findings}

The distribution of clinicopathological findings is shown in Table 1. Most of the 10 OLP patients (15 sites) showed the main lesion involving the buccal mucosa (14 sites), followed by the gingiva ( 1 site). Most of the 9 patients with OLLs (16 sites) likewise showed the main lesion involving the buccal mucosa (12 sites), followed by the gingiva (4 sites). The validity of the clinical diagnosis of OLP was consistent at 5 sites, but the remaining included 4 carcinomas and 1 instance of leukoplakia. For OLLs, OLP, malignant tumor and leukoplakia were 6, 2 and 1, respectively. In terms of chief complaints, $10.0 \%$ and $33.3 \%$ with OLP and $33.3 \%$ and $11.1 \%$ with OLLs presented with pain and discomfort, respectively. Figure 1 shows representative macroscopic pictures of OLP (Figure 1(a)) and OLLs (Figure 1(b)). Both show similar clinical conditions with a mixture of white striae and erosions. No specific macroscopic findings were found for either disease. Representative case where treatment was successful is shown in Figure 1(e) and Figure 1(f). OLP patient without dental metal allergies of the left buccal mucosa with white patch and erosion pre- (e) and post-treatment (f). Most of the white patch and erosions disappeared after treatment.

\subsection{Histopathological Findings}

Figure 1 shows representative histopathological pictures of OLP (Figure 1(c)) and OLLs (Figure 1(d)). In OLP, at sites where basal cells were lost due to degeneration, spinous cells were in direct contact with band-like lymphocyte infiltration in the subepithelial connective tissue (Figure 1(c)). OLLs showed slight band-like or diffuse infiltration of lymphocytes into subepithelial connective tissue, although no loss of basal cells was observed (Figure 1(d)). In high-power fields (Figures 2(a)-(c)), melanin pigmentation just below the basal membrane was observed in all 10 OLP specimens (100\%) and 2 of 9 OLL specimens (22\%).

\subsection{Immunohistochemical and Papanicolaou Findings}

The results of immunohistochemical staining are shown in Figure 3. For CK19 (Figure 3(a)), positive findings were continuously expressed in the basal layer of normal mucosa. In OLLs, CK19-positive findings were discontinuous in the 

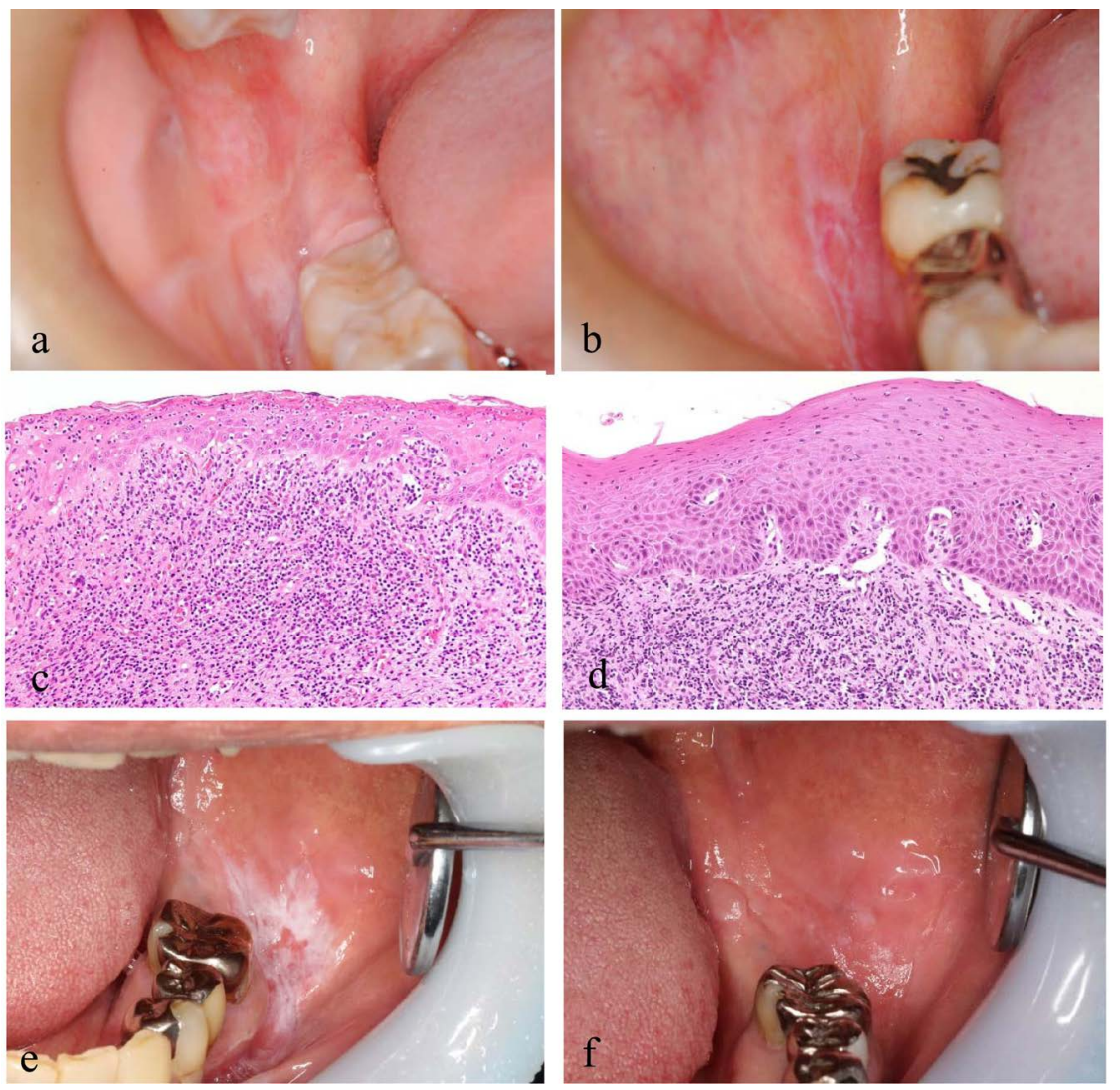

Figure 1. Gross and histopathological findings of oral lichen planus (OLP) and oral lichenoid lesions (OLLs). (a, b): OLP (a) and OLLs (b) of the right buccal mucosa with white striae and erosive lesion; (c): Liquefaction degeneration in the basement membrane and loss of basal cells in OLP (Hematoxyline and Eosin staining, x20); (d): No degeneration in the basement membrane and vascularity in basal cells in OLLs (Hematoxyline and Eosin staining, x20); (e, f): OLP patient without dental metal allergies of the left buccal mucosa with white patch and erosion pre- (e) and post-treatment (f).
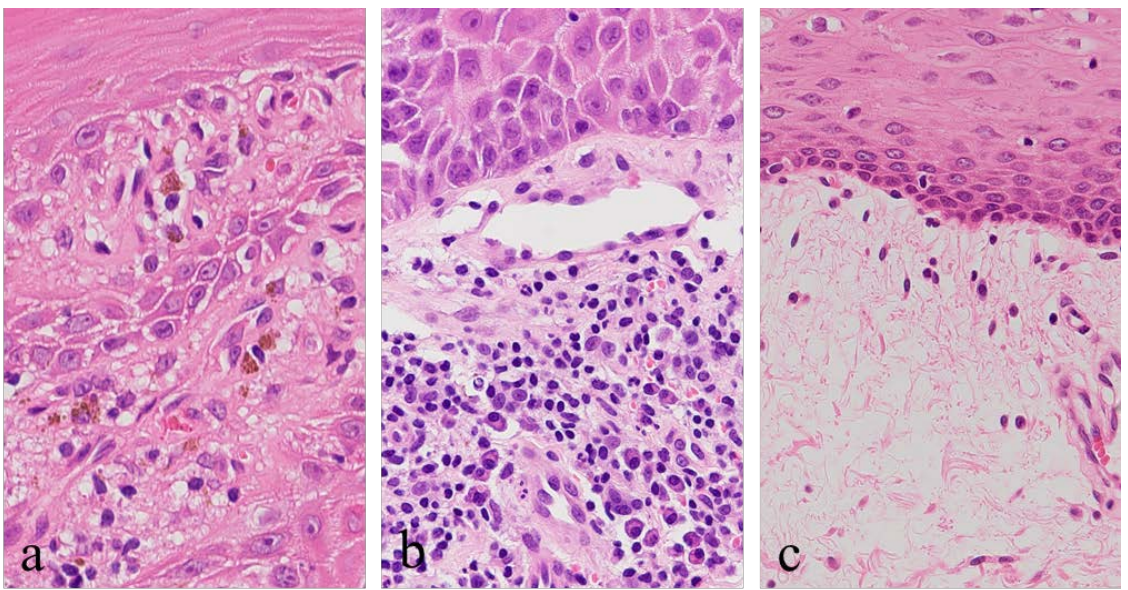

Figure 2. High power field of basal layer and subepithelial tissue of oral lichen planus (OLP) and oral lichenoid lesions (OLLs) and normal tissue (Hematoxyline and Eosin staining, x20) (a): Melanin pigment and melanophores are scattered in OLP; (b): Many mixed inflammatory cells are infiltrated, but no melanophores in OLLs; (c): Very few lymphocytes are scattered in normal mucosa. 


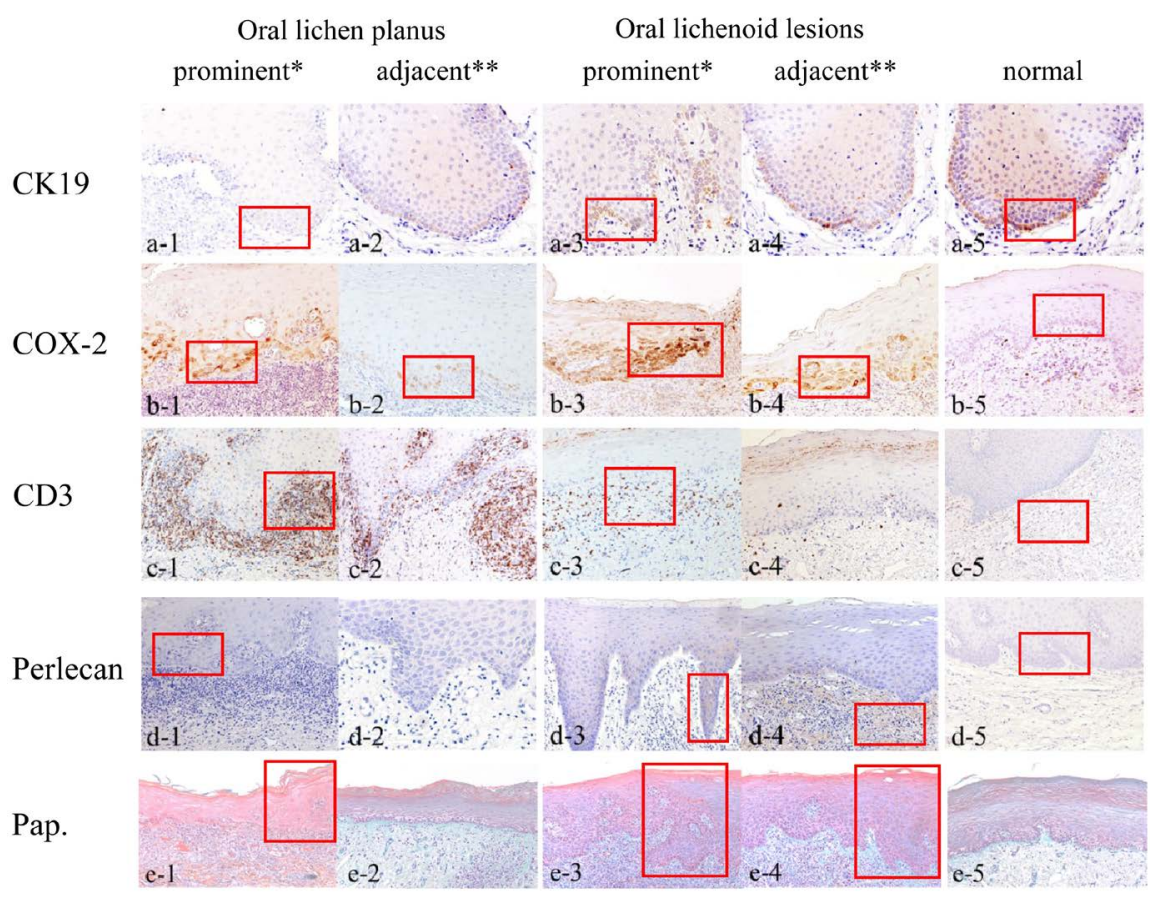

Figure 3. Photographs of each representative immunohistochemical and Papanicolaou staining results. ${ }^{*}$ : the most prominent lesion; ${ }^{* *}$ : an adjacent area of normal mucosa; Pap.: Papanicolaou staining; Main different findings were highlighted by red circle. $(a(1-5), b(1-5)), c(1-5)), d(1-5))$ shows CK19, COX-2, CD3 and Perlecan staining in oral lichen planus (OLP) and oral lichenoid lesions (OLLs), respectively. CK19 showed almost no positive cells at prominent area in OLP and scattered in OLLs. COX-2 reaction was apparent at prominent and adjacent area in OLLs. Many CD3 positive cells were appeared directly under spinous layer in OLP. Perlecan reacted epithelial cells and stroma in OLLs. (e(1-5)) present Papanicolaou staining. Thickening of the spinous layer was observed in all areas of OLLs.

prominent area and almost continuous in the site adjacent the lesion. As for CK19, no staining was noted in contact with the basement membrane in the prominent area and weakly positive in the site adjacent the lesion in OLP. Both were reduced compared to OLLs (Figures $3(\mathrm{a}-1,2)$ ).

For COX-2 (Figure 3(b)), positive cells were slightly scattered in the stroma of normal mucosa. In OLP, COX-2 was expressed in the basal to deep spinous layers of the prominent area and scattered in the deep spinous layer at the site adjacent to the lesion (Figures $3(\mathrm{~b}-1,2)$ ). In OLLs, numerous positive cells were seen in the basal to spinous layers of the prominent area and moderate expression was identified in the basal to deep spinous layers at the site adjacent to the lesion. Many positive cells were observed in the submucosal connective tissue in OLP and OLLs (Figure 3(b-1), Figure 3(b-3)).

For CD3 (Figure 3(c)), it exhibited some positive findings in normal mucosa. OLP showed positive findings in most of the band-like infiltrated lymphocytes, and invasion into the epithelium was also observed. In the site adjacent to the lesion, positive cells appeared in a slightly band-like infiltration (Figures $3(\mathrm{c}-1$, 2)). In OLLs, positive cells were diffusely present at the subepithelial junction 
and were scattered at the site adjacent to the lesion (Figures 3(c-3,4)).

Perlecan was negative in normal mucosa. In OLP, negative results were seen in both the prominent area and the site adjacent to the lesion (Figures 3(d-1, $2)$ ). In OLLs, epithelial cells were mildly positive in the prominent area and were slightly positive in basal cells at the site adjacent the lesion. Strong positive reactions were shown in submucosal tissue at the site adjacent the lesion (Figures $3(\mathrm{~d}-3,4))$. Many Ki-67-positive cells were seen in both OLP and OLLs at prominent areas and the adjacent sites (data not shown).

Comparing the distributions of positive expressions for CK19 and CD3, CD3-positive cells showed a band-like distribution in contact with the spinous-layer cells that lost CK19 expression in OLP (Figure 3(a-1), Figure 3(c-1)). In OLLs, more CK19-positive cells were found in contact with the stroma, and CD3-positive cells were scattered in the stroma (Figure 3(a-3), Figure 3(c-3)).

Increased keratinization, which stains orange, was observed at the prominent area and adjacent sites of both OLP and OLLs (Figures $3(\mathrm{e}-1,2,3,4)$ ). Thickening of the spinous layer was observed in all areas of OLLs.

\subsection{Statistical Analysis}

Statistical results are presented in Figures 4-6. For CK19, positive cells were significantly found in OLLs at both the prominent area and site adjacent to the lesion comparison with those of OLP's ( $p<0.05$, Figure 4$)$. The number of COX-2-positive cells was significantly higher in spinous $(p<0.05)$ and basal layers $(p<0.01)$ in OLLs of the prominent area. Conversely, OLP showed significantly more positive reaction than OLLs at the site adjacent to the lesion ( $p<$ 0.05, Figure 5). Concerning Ki-67, no significant difference was seen between OLP and OLLs in either prominent areas or sites adjacent to the lesion (data not shown). Figure 6 shows the results for thickness of the epithelium from the

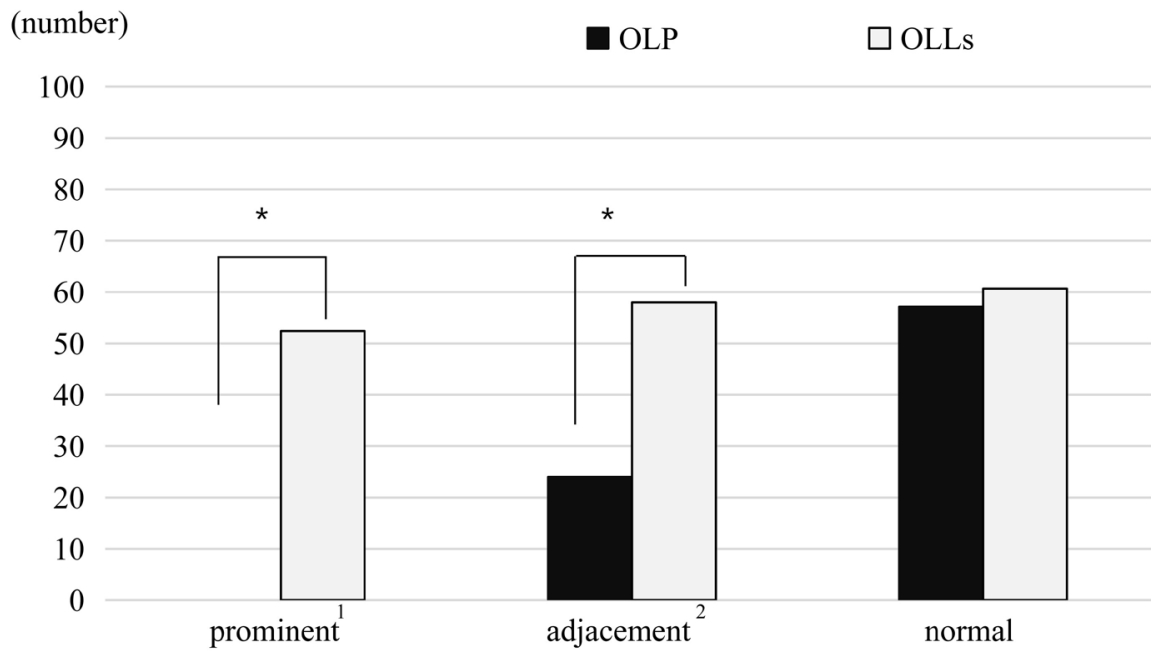

Figure 4. Results of CK19 immunohistochemical staining. Black, light gray bars indicate oral lichen planus (OLP) and oral lichenoid lesions (OLLs), respectively. ${ }^{1}$ : the most prominent lesion; ${ }^{2}$ : an adjacent area of normal mucosa; ${ }^{*}: p<0.05$. 
(number)

50

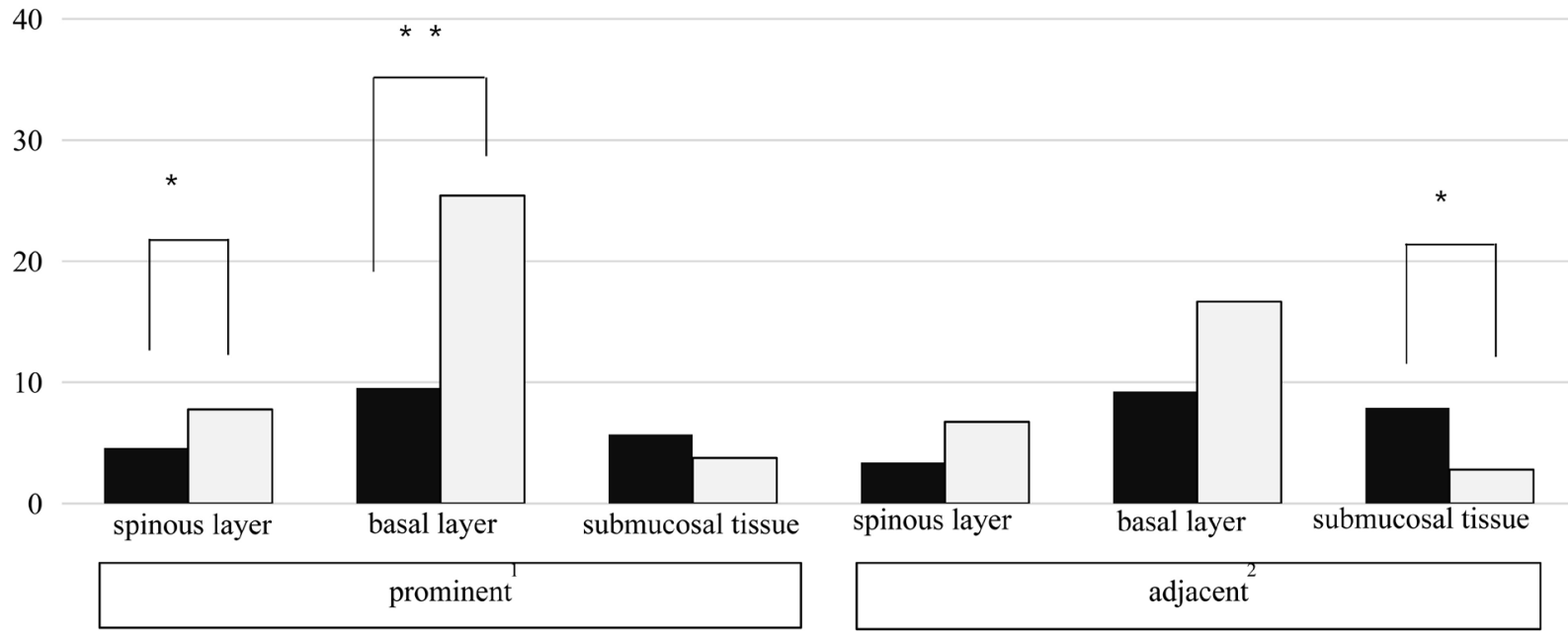

Figure 5. Results of COX-2 immunohistochemical staining. Black, light gray bars indicate oral lichen planus (OLP) and oral lichenoid lesions (OLLs), respectively. ${ }^{1}$ : the most prominent lesion; ${ }^{2}$ : an adjacent area of normal mucosa; ${ }^{*}: p<0.05 ;{ }^{* *}: p<0.01$.

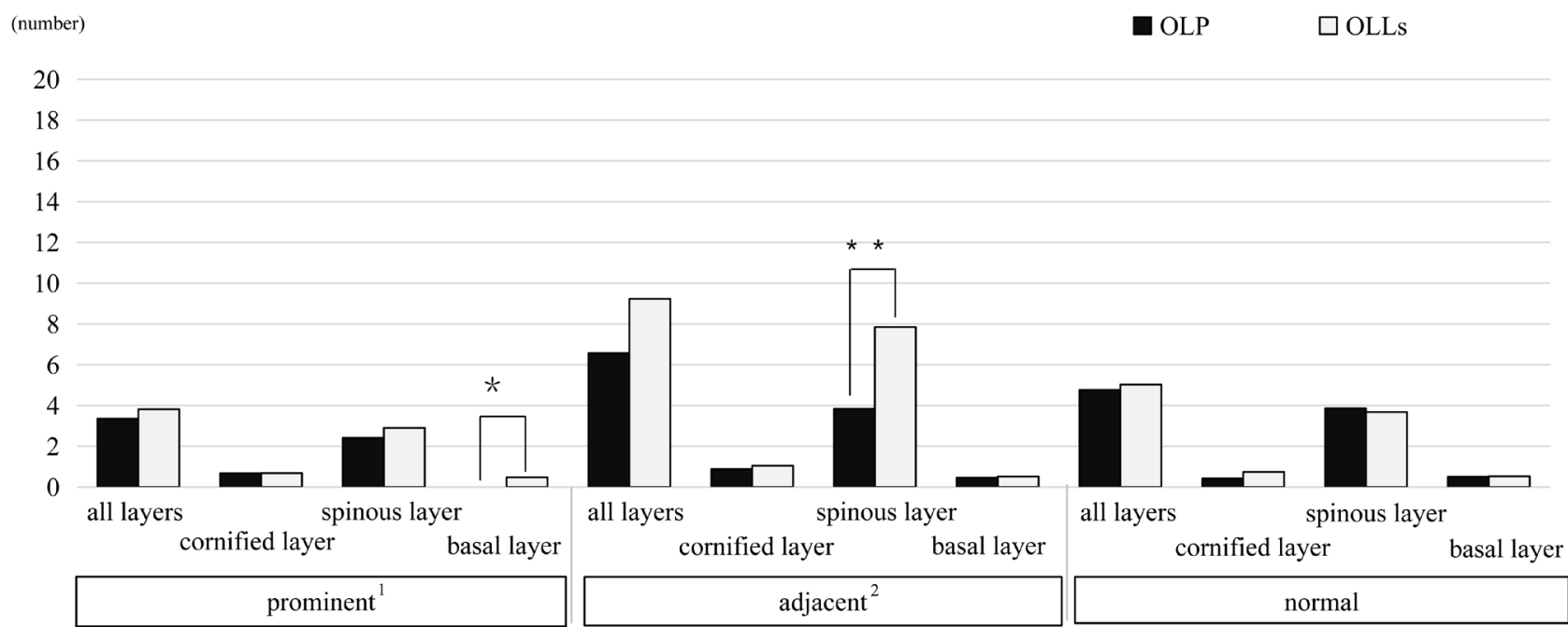

Figure 6. Result of epithelial layer thickness by Papanicolaou staining. Black, light gray bars indicate oral lichen planus (OLP) and oral lichenoid lesions (OLLs), respectively. ${ }^{1}$ : the most prominent lesion; ${ }^{2}$ : an adjacent area of normal mucosa; ${ }^{*}: p<0.05 ;{ }^{* *}: p<$ 0.01 .

results of Papanicolaou staining. Spinous and basal layers at both the site adjacent to the lesion and the prominent area in OLLs were thicker than those in OLP $(p<0.05)$.

\section{Discussion}

Lichenoid reactions are observed in various lesions, but the malignant transformation rate of OLLs should be noted to be higher than that of OLP [9]. Ex- 
amining these histopathological differential findings is thus of great significance.

Cytokeratins are keratin-containing intermediate filament proteins found in the intracytoplasmic cytoskeleton of epithelial tissue. Among the 20 epithelial cytokeratins that have been identified, CK19 is the lowest molecular weight acidic keratin $(40 \mathrm{kDa})$ and is a specific cytoskeletal structure of simple epithelium and the basal cells of stratified squamous epithelium [21]. In the OLP lesion, T-cell infiltration was induced just below the epithelium after recognition of an unknown antigen and involved a cell-mediated immune response and might have attacked CK19-positive basal cells in the present study. On the other hand, in OLLs, basal cells remained discontinuous, and have been speculated to facilitate histopathological differentiation. OLP is a lymphocyte-mediated immunological disorder in which the basal cells seem to be targeted by $\mathrm{T}$ lymphocytes [22] [23] [24] [25]. Basal cells disappeared in areas of OLLs in this study, and common events in the lichenoid tissue reaction are speculated to include activation of dendritic cells and keratinocytes, and recruitment and activation of $\mathrm{T}$ cells, followed by cytotoxic damage to keratinocytes with release of keratinocyte antigens [26]. The positive findings for CD3 and CK19 in OLP and OLLs were consistent with the above speculation in the present study.

$\mathrm{Ki}-67$ is often used as an adjunct marker to assess the proliferative activity of potentially malignant lesions [27]. In the present study, no significant difference was seen between OLP and OLLs with Ki-67-positive rates in either the prominent areas or adjacent sites. This result was concordant with the results of a previous study [28]. Because both lesions represent chronic inflammation, the increase in Ki-67-positive cells might show that the epithelium is required for enhanced proliferation and healing [22]. Concerning epithelial thickness as determined by Papanicolaou staining, spinous and basal layers at the adjacent site and prominent area in OLLs were thicker than those in OLP. This implied that growth of the epithelium was promoted by something like a chronic regeneration reaction or epithelial growth factor in OLLs, despite the lack of significant difference in Ki-67 positivity between OLP and OLLs.

Perlecan, a heparan sulfate proteoglycan of about $470 \mathrm{kDa}$ in size, is one of the major basement membrane macromolecules and plays an important role in cellular growth, differentiation, adhesion, and motility through its interactions with growth factors and cytokines [19] [20]. Recently, perlecan has been localized in the intercellular space of the oral epithelium and is overexpressed in dysplastic epithelial cells and deposited in their interepithelial space, resulting in the histological finding of reduced of cellular cohesion [20]. OLLs show mild to moderate expression in the basal to spinous layers of epithelial cells of the lesion. Positive expression was shown in the adjacent area and weak to moderate positivity was seen in subepithelial tissue. Conversely, negative results were seen for epithelial cells in the prominent and adjacent area, and in the stroma of the adjacent area in OLP of the present study. For epithelial cells in OLLs, perlecan is speculated to be synthesized by germ cells with parabasal cell-like appearances, as major constituent cells of epithelial dysplasia, and is deposited in the intercellular space 
of dysplastic epithelial cells to facilitate their proliferation [20]. Another possible function of intraepithelial perlecan was considered to be in providing a space for the migration of intraepithelial cells, such as lymphocytes and macrophages (including Langerhans cells), which are usually distributed in the epithelial layer of the oral mucosa and are thought to patrol for immune stimulations from the oral cavity [29]. Concerning perlecan in stroma, the myxoid or edematous matrices of immature granulation tissues were shown to be immunopositively simultaneous with epithelial cells [19]. Perlecan is expressed in the process of tissue remodeling of inflammatory lesions [30]. Tissue regeneration involving perlecan is speculated to be taking place in the stroma of OLLs, but the relationship with the acquisition of neoplastic characteristics of epithelial cells needs further investigation.

Cyclooxygenases (or prostaglandin $\mathrm{H}$ synthases), commonly referred to as COXs, are a family of myeloperoxidases located on the luminal side of the endoplasmic reticulum and nuclear membrane [31] and catalyze the rate-limiting step of prostaglandin biosynthesis from arachidonic acid [31]. The COX-2 enzyme is often expressed during inflammation. COX-2 aids in tissue repair, angiogenesis, cell proliferation and differentiation, but chronic, persistent inflammation as noted in OLP and OLLs can lead to detrimental effects [32]. In addition, inflammation is strongly associated with carcinogenesis, including the development and progression of oral cancer [33] [34]. Further, COX-2 expression has been correlated with higher grades of oral epithelial dysplasia and could represent an early event in oral carcinogenesis [35] [36]. Several studies, including that of Arreaza, et al. [32], found a higher COX-2 expression in OLP than in OLLs, and Chankong, et al. [37] observed a direct correlation between COX-2 expression in OLP and the clinical severity of this pathology. Conversely, Cortes et al. found higher expression of COX-2 in OLLs than in OLP [38]. In the present study, the number of COX-2-positive cells in epithelium was significantly higher in OLLs of the prominent area. However, OLP showed significantly more positive reactions than OLLs at the adjacent sites. COX-2 overexpression by epithelial cells in OLLs could inferred oncogenic alterations, because was described to modulate cell proliferation and apoptosis against oncogenic alterations [39] [40] [41].

Accordingly, COX-2 and perlecan were found in the basal to spinous layers of the prominent area and adjacent site, and of the prominent area in OLLs, respectively, in the present study. These findings suggest the acquisition of neoplastic characteristics in the epithelial cells of OLLs [35]. Conversely, of all OPMDs, malignant transformation of OLP has been described as the most controversial [38]. The authors also concluded that this finding suggested a different etiology and molecular pathophysiological pathways for OLP and OLLs. Patil, et al. [42] and Fitzpatrick [43] also observed features of dysplasia in OLP and OLL in their case series, reiterating the malignant potential of OLP and OLL.

It is therefore very important for the pathologist to differentiate OLP from epithelial dysplasia with lichenoid features, as the distinction has significant impli- 
cations for diagnosis, research and controversies surrounding OLP with regard to its malignant potential.

\section{Conclusions}

The following could be concluded in the present study:

1) Almost no basal cells were noted in the prominent area in OLP and were significantly reduced compared to OLLs.

2) COX-2 was found in the prominent area in OLLs with significance.

3) OLLs showed mild to moderate expression for perlecan in the basal to spinous layers and in subepithelial tissue.

4) Although there are restrictions, these suggested the possibility of helping to distinguish between OLP and OLLs.

\section{Acknowledgements}

This work was supported by JSPS KAKENHI Grant Number 18K07000.

\section{Conflicts of Interest}

The authors declare no conflicts of interest regarding the publication of this paper.

\section{References}

[1] Petruzzi, M., De Benedittis, M., Grassi, R., Cassano, N., Vena, G. and Serpico, R. (2002) Oral Lichen Planus: A Preliminary Clinical Study on Treatment with Tazarotene. Oral Diseases, 8, 291-295. https://doi.org/10.1034/j.1601-0825.2002.02833.x

[2] Pindborg, J.J., Reichart, P.A., Smith, C.V. and van der Waa, I. (1997) Histological Typing of Cancer and Precancer of the Oral Mucosa. 2nd Edition, Springer, Berlin, Heidelberg, 30. https://doi.org/10.1007/978-3-642-60592-5

[3] Cawson, R.A. and Odell, E.W. (1998) Diseases of the Oral Mucosa: Non-Infective Stomatitis. In: Cawson, R.A. and Odell, E.W., Eds., Cawson's Essentials of Oral Pathology and Oral Medicine, 6th Edition, Churchill Livingstone, London, 187-191.

[4] Hasegawa, K., Sakamaki, H., Higuchi, M., Suemitsu, M., Chieko, T., Ito, K. and Kayo, K. (2018) Histomorphometric Evaluation of Intraepithelial Papillary Capillaries in Oral Lichen Planus: A Histopathological Study. Journal of Dermatology Research and Therapy, 4, 58. https://doi.org/10.23937/2469-5750/1510058

[5] Bombeccari, G. P., Giannì, A. B. and Spadari, F. (2017) Immunoexpression of Cytokeratin-19 in the Oral Lichen Planus and Related Oral Squamous Cell Carcinoma. Annali Di Stomatologia, 8, 104-109.

[6] Liu, Y., Liu, G., Liu, Q., Tan, J., Hu, X., Wang, J. and Wang, X. (2017) The Cellular Character of Liquefaction Degeneration in Oral Lichen Planus and the Role of Interferon Gamma. Journal of Oral Pathology and Medicine, 46, 1015-1022. https://doi.org/10.1111/jop.12595

[7] Mattila, R., Alanen, K. and Syrjänen, S. (2007) Immunohistochemical Study on Topoisomerase IIa, Ki-67 and Cytokeratin-19 in Oral Lichen Planus Lesions. Archives of Dermatological Research, 298, 381-388. https://doi.org/10.1007/s00403-006-0711-z 
[8] Eisenberg, E. and Krutchkoff, D.J. (1992) Lichenoid Lesions of Oral Mucosa. Oral Surgery, Oral Medicine, Oral Pathology, 73, 699-704. https://doi.org/10.1016/0030-4220(92)90013-G

[9] Kamath, V., Setlur, K. and Yerlagudda, K. (2015) Oral Lichenoid Lesions-A Review and Update. Indian Journal of Dermatology, 60, 102. https://doi.org/10.4103/0019-5154.147830

[10] Reibel, J., Tilakaratne, W., Gale, N., Westra, W., Hille, J., Williams, M. and Zain, R. (2017) Oral Potentially Malignant Disorders and Oral Epithelial Dysplasia. In: El-Naggar, A.K., Chan, J.K.C., Grandis, J.R., Takata, T. and Slootweg, P.J., Eds., WHO Classification of Head and Neck Tumours, 4th Edition, International Agency for Research on Cancer (IARC), Lyon, 112-115.

[11] El-Naggar, A.K. and Reichart, P. (2015) Proliferative Verrucous Leukoplakia and Precancerous Conditions. In: Barnes, L., Eveson, J.W., Reichart, P. and Sidransky, D., Eds., World Health Organization Classification of Tumours, Pathology \& Genetics Head and Neck Tumours, International Agency for Research on Cancer (IARC), Lyon, 180-181.

[12] Thornhill, M.H., Sankar, V., Xu, X.J., Barrett, A.W., High, A.S., Odell, E.W. and Farthing, P.M. (2006) The Role of Histopathological Characteristics in Distinguishing Amalgam-Associated Oral Lichenoid Reactions and Oral Lichen Planus. Journal of Oral Pathology and Medicine, 35, 233-240.

https://doi.org/10.1111/j.1600-0714.2006.00406.x

[13] Alrashdan, M.S, Cirillo, N. and Mccullough, M. (2016) Oral Lichen Planus: A Literature Review and Update. Archives of Dermatological Research, 308, 539-551. https://doi.org/10.1007/s00403-016-1667-2

[14] Al-Hashimi, I., Schifter, M., Lockhart, P.B., Wray, D., Brennan, M., Migliorati, C.A. and van der Waal, I. (2007) Oral Lichen Planus and Oral Lichenoid Lesions: Diagnostic and Therapeutic Considerations. Oral Surgery, Oral Medicine, Oral Pathology, Oral Radiology and Endodontology, 103, S25.e1-S25.e12.

https://doi.org/10.1016/j.tripleo.2006.11.001

[15] Schmidt-Westhausen, A.M. (2020) Oral Lichen Planus and Lichenoid Lesions: What's New? Quintessence International, 51, 156-161. https://doi.org/10.3290/j.qi.a43868

[16] Andreasen, J.O. (1968) Oral Lichen Planus. 1. A Clinical Evaluation of 115 Cases. Oral Surgery, Oral Medicine, Oral Pathology, 25, 31-42.

https://doi.org/10.1016/0030-4220(68)90194-1

[17] Brant, J.M.C., Vasconcelos, A.C. and Rodrigues, L.V. (2008) Role of Apoptosis in Erosive and Reticular Oral Lichen Planus Exhibiting Variable Epithelial Thickness. Brazilian Dental Journal, 19, 179-185. http://dx.doi.org/10.1590/S0103-64402008000300001

[18] Van der Meij, E.H., Mast, H. and van der Waal, I. (2007) The Possible Premalignant Character of Oral Lichen Planus and Oral Lichenoid Lesions: A Prospective Five-Year Follow-up Study of 192 Patients. Oral Oncology, 43, 742-748. https://doi.org/10.1016/j.oraloncology.2006.09.006

[19] Yamazaki, M., Cheng, J., Hao, N., Takagi, R., Jimi, S., Itabe, H. and Saku, T. (2004) Basement Membrane-Type Heparan Sulfate Proteoglycan (Perlecan) and Low-Density Lipoprotein (LDL) Are Co-Localized in Granulation Tissues: A Possible Pathogenesis of Cholesterol Granulomas in Jaw Cysts. Journal of Oral Pathology and Medicine, 33, 177-184. https://doi.org/10.1111/j.0904-2512.2004.00087.x

[20] Ikarashi, T., Ida-Yonemochi, H., Ohshiro, K., Cheng, J. and Saku, T. (2004) Intra- 
epithelial Expression of Perlecan, a Basement Membrane-Type Heparan Sulfate Proteoglycan Reflects Dysplastic Changes of the Oral Mucosal Epithelium. Journal of Oral Pathology and Medicine, 33, 87-95. https://doi.org/10.1111/j.1600-0714.2004.00026.x

[21] Masai, K., Nakagawa, K., Yoshida, A., Sakurai, H., Watanabe, S., Asamura, H. and Tsuta, K. (2014) Cytokeratin 19 Expression in Primary Thoracic Tumors and Lymph Node Metastases. Lung Cancer, 86, 318-323.

https://doi.org/10.1016/j.lungcan.2014.09.018

[22] Piattelli, A., Carinci, F., Iezzi, G., Perrotti, V., Goteri, G., Fioroni, M. and Rubini, C. (2007) Oral Lichen Planus Treated with 13-cis-Retinoic Acid (Isotretinoin): Effects on the Apoptotic Process. Clinical Oral Investigations, 11, 283-288.

https://doi.org/10.1007/s00784-007-0117-0

[23] Hirota, M., Ito, T., Okudela, K., Kawabe, R., Yazawa, T., Hayashi, H. and Kitamura, H. (2002) Cell Proliferation Activity and the Expression of Cell Cycle Regulatory Proteins in Oral Lichen Planus. Journal of Oral Pathology \& Medicine, 31, 204-212. https://doi.org/10.1034/j.1600-0714.2002.310403.x

[24] Karatsaidis, A., Schreurs, O., Helgeland, K., Axéll, T. and Schenck, K. (2003) Erythematous and Reticular Forms of Oral Lichen Planus and Oral Lichenoid Reactions Differ in Pathological Features Related to Disease Activity. Journal of Oral Pathology and Medicine, 32, 275-281. https://doi.org/10.1034/j.1600-0714.2003.00134.x

[25] Zhao, Z.Z., Savage, N.W., Sugerman, P.B. and Walsh, L.J. (2002) Mast Cell/T Cell Interactions in Oral Lichen Planus. Journal of Oral Pathology and Medicine, 31, 189-195. https://doi.org/10.1034/j.1600-0714.2002.310401.x

[26] Dutz, J.P. (2009) T-Cell-Mediated Injury to Keratinocytes: Insights from Animal Models of the Lichenoid Tissue Reaction. Journal of Investigative Dermatology, 129, 309-314. https://doi.org/10.1038/jid.2008.242

[27] Pigatti, F.M., Taveira, L.A. de A. and Soares, C.T. (2015) Immunohistochemical Expression of Bcl-2 and Ki-67 in Oral Lichen Planus and Leukoplakia with Different Degrees of Dysplasia. International Journal of Dermatology, 54, 150-155. https://doi.org/10.1111/ijd.12279

[28] Acay, R.R., Felizzola, C.R., Soares de Araújo, N. and Machado de Sousa, S.O. (2006) Evaluation of Proliferative Potential in Oral Lichen Planus and Oral Lichenoid Lesions Using Immunohistochemical Expression of p53 and Ki67. Oral Oncology, 42, 475-480. https://doi.org/10.1016/j.oraloncology.2005.09.012

[29] Walton, L.J., Macey, M.G., Thornhill, M.H. and Farthing, P.M. (1998) Intraepithelial Subpopulations of T Lymphocytes and Langerhans Cells in Oral Lichen Planus. Journal of Oral Pathology \& Medicine, 27, 116-123. https://doi.org/10.1111/j.1600-0714.1998.tb01926.x

[30] Murata, M., Hara, K. and Saku, T. (1997) Dynamic Distribution of Basic Fibroblast Growth Factor during Epulis Formation: An Immunohistochemical Study in an Enhanced Healing Process of the Gingiva. Journal of Oral Pathology and Medicine, 26, 224-232. https://doi.org/10.1111/j.1600-0714.1997.tb01228.x

[31] Sobolewski, C., Cerella, C., Dicato, M., Ghibelli, L. and Diederich, M. (2010) The Role of Cyclooxygenase-2 in Cell Proliferation and Cell Death in Human Malignancies. International Journal of Cell Biology, 2010, Article ID: 215158. https://doi.org/10.1155/2010/215158

[32] Arreaza, A.J., Rivera, H. and Correnti, M. (2014) Expression of COX-2 and BCL-2 in Oral Lichen Planus Lesions and Lichenoid Reactions. Ecancermedicalscience, 8, 
Article no. 411. https://doi.org/10.3332/ecancer.2014.411

[33] Sarode, G.S., Sarode, S.C., Patil, A., Anand, R., Patil, S.G., Rao, R.S. and Augustine, D. (2015) Inflammation and Oral Cancer: An Update Review on Targeted Therapies. The Journal of Contemporary Dental Practice, 16, 595-602. https://doi.org/10.5005/jp-journals-10024-1727

[34] Sarode, S.C., Sarode, G.S. and Patil, A. (2014) Therapeutic Aspects of the Inflammation Mediated Oral Carcinogenesis. Oral Oncology, 50, e13-e14. https://doi.org/10.1016/j.oraloncology.2013.12.026

[35] Shibata, M., Kodani, I., Osaki, M., Araki, K., Adachi, H., Ryoke, K. and Ito, H. (2005) Cyclo-Oxygenase-1 and -2 Expression in Human Oral Mucosa, Dysplasias and Squamous Cell Carcinomas and Their Pathological Significance. Oral Oncology, 41, 304-312. https://doi.org/10.1016/j.oraloncology.2004.09.009

[36] Sanketh, D.S., Kumari, K., Rao, R.S., Haragannavar, V.C., Sarode, S.C., Sarode, G.S. and Patil, S. (2019) Expression of Ki-67, p53, a-SMA and COX-2 in Lichen Planus and Related Lesions: A Pilot Study. Journal of Oral Biology and Craniofacial Research, 9, 230-235. https://doi.org/10.1016/j.jobcr.2018.02.003

[37] Chankong, T., Chotjumlong, P., Sastraruji, T., Pongsiriwet, S., Iamaroon, A. and Krisanaprakornkit, S. (2016) Increased Cyclooxygenase 2 Expression in Association with Oral Lichen Planus Severity. Journal of Dental Sciences, 11, 238-244. https://doi.org/10.1016/j.jds.2015.12.002

[38] Cortés-Ramírez, D.A., Rodrguez-Tojo, M.J., Gainza-Cirauqui, M.L., Martnez-Conde, R. and Aguirre-Urizar, J.M. (2010) Overexpression of Cyclooxygenase-2 as a Biomarker in Different Subtypes of the Oral Lichenoid Disease. Oral Surgery, Oral Medicine, Oral Pathology, Oral Radiology and Endodontology, 110, 738-743. https://doi.org/10.1016/j.tripleo.2010.08.005

[39] Lynch, P.M. (2001) COX-2 Inhibition in Clinical Cancer Prevention. Oncology, 15, 21-26.

[40] Arber, N. (2008) Cyclooxygenase-2 Inhibitors in Colorectal Cancer Prevention: Point. Cancer Epidemiology Biomarkers and Prevention, 17, 1852-1857.

[41] Arun, B. and Goss, P. (2004) The Role of COX-2 Inhibition in Breast Cancer Treatment and Prevention. Seminars in Oncology, 31, 22-29. https://doi.org/10.1053/j.seminoncol.2004.03.042

[42] Patil, S., Rao, R.S., Sanketh, D.S. and Warnakulasuriya, S. (2015) Lichenoid Dysplasia Revisited-Evidence from a Review of Indian Archives. Journal of Oral Pathology \& Medicine, 44, 507-514. https://doi.org/10.1111/jop.12258

[43] Fitzpatrick, S.G., Honda, K.S., Sattar, A. and Hirsch, S.A. (2014) Histologic Lichenoid Features in Oral Dysplasia and Squamous Cell Carcinoma. Oral Surgery, Oral Medicine, Oral Pathology and Oral Radiology, 117, 511-520. https://doi.org/10.1016/j.oooo.2013.12.413 